\title{
Extracorporeal Membrane Oxygenation as a Bridge between Transfer and Perioperative Periods in Refractory Cardiogenic Shock Secondary to a Large Left Atrial Myxoma
}

\author{
Lijun Tian, MD, Suyan Zhang, MD, Junxian Xu, MD, Xudong Han, MD \\ Department of Critical Care Medicine, Nantong Third People's Hospital, Nantong University, Nantong, China
}

\section{ABSTRACT}

A 63-year-old man was admitted for acute left heart failure after doing farm work. He rapidly developed refractory cardiogenic shock due to a large left atrial myxoma which was found by bedside echocardiography. Venoarteriovenous extracorporeal membrane oxygenation (ECMO) was performed immediately, and the patient was transferred for further surgery with a good outcome. Therefore, timely echocardiographic evaluation and surgical removal of myxomas is recommended, and ECMO could be used as a bridge between the transfer and perioperative period.

\section{INTRODUCTION}

Cardiac myxomas are rare, and approximately $75 \%$ of them are known to develop in the left atrium. Clinical symptoms of cardiac myxomas vary from unapparent to lethal complications such as embolisms, acute heart failure, or even sudden death [Ali 2018]. Timely diagnosis and urgent surgical treatment are crucial [Wedin 2020]; however, there are still a number of patients who die of severe heart failure preoperatively [Layton 2013]. Here, we report the case of a patient with a large left atrial myxoma that was asymptomatic until the acute onset of heart failure along with refractory shock. Extracorporeal membrane oxygenation $(\mathrm{ECMO})$ was used as a bridge between his transfer and surgery.

\section{CASE REPORT}

A 63-year-old male patient was admitted to the local hospital for acute left heart failure after doing farm work. He

Received November 30,2020; received in revised form fanuary 9, 2021; accepted Fanuary 10, 2021.

This work was supported by the Nantong Science and Technology Bureau [grant numbers XG202003-3 and FC2020054]; Nantong Municipal Commission of Health and Family Planning [grant numbers MA2020016, MA2020017, MA2020036].

Correspondence: Xudong Han, MD, Department of Critical Care Medicine, Nantong Third People's Hospital, Nantong University, 60 Mid-Youth Road, Nantong, 226006, China; (e-mail: banxudong9610@163.com). suffered from severe hypoxemia along with cardiogenic shock. He was intubated 12 hours after treatment with diuresis,
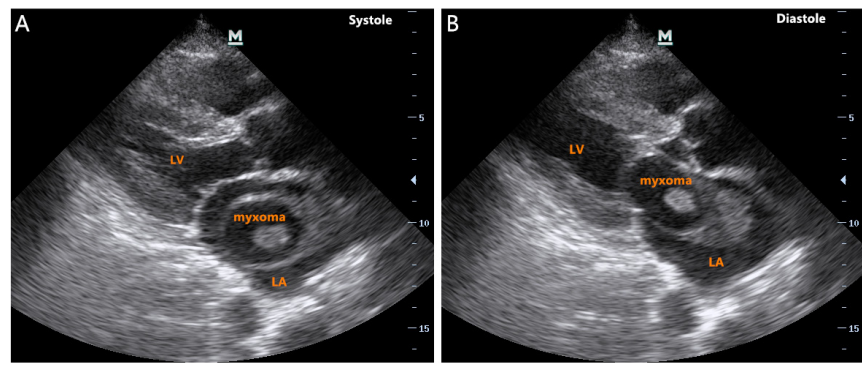

Figure 1. Echocardiogram revealing an "evil eye" floating in the left atrium. (A) Systolic; (B) Diastole.
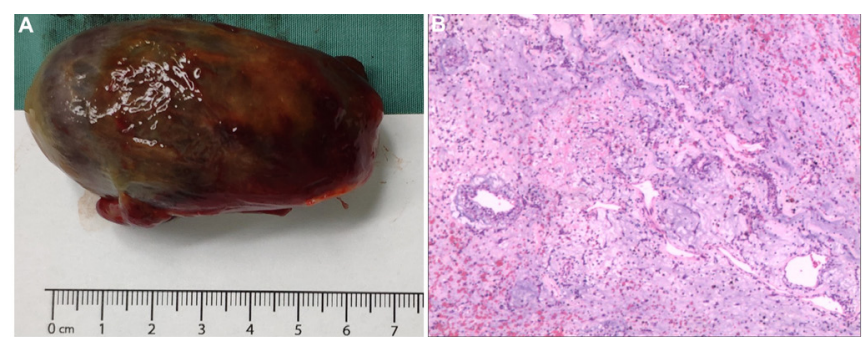

Figure 2. (A) Surgical specimen of the mass (diameter: $75 \mathrm{~mm}$ ); (B) Hematoxylin and eosin staining.

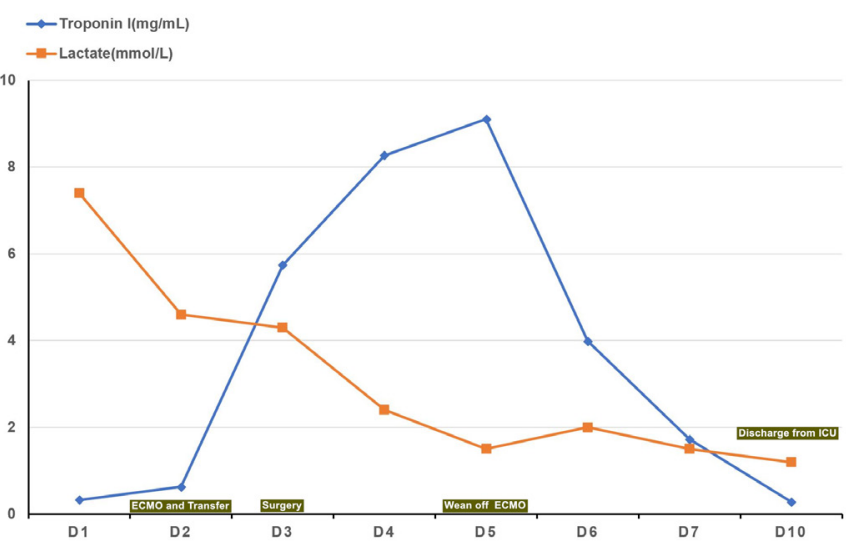

Figure 3. Changes in troponin I and lactate. 


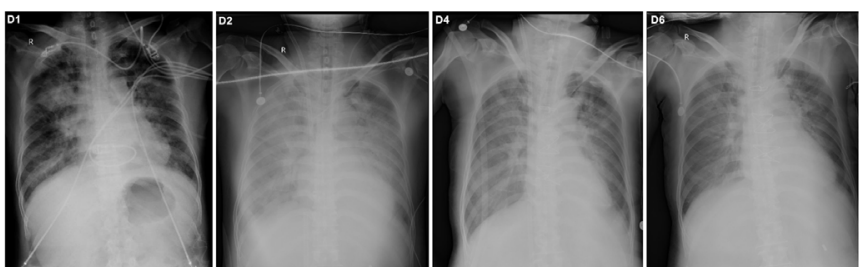

Figure 4. Series of chest $\mathrm{x}$-rays.

inotropes, vasodilators, and non-invasive positive pressure ventilation. When our team arrived on day 2 , his blood pressure was maintained at $80 / 50 \mathrm{mmHg}$ with high-dose inotropic support (noradrenaline [100 ug/min], pituitrin [2 U/h], and dobutamine $[20 \mathrm{ug} / \mathrm{kg} / \mathrm{min}]$ ), and his $\mathrm{PaO} 2 / \mathrm{FiO} 2(\mathrm{P} / \mathrm{F})$ ratio was only $80 \mathrm{mmHg}$. It is worth noting that his blood pressure considerably fluctuated with heart rate. A bedside echocardiography showed a giant anomalous vegetation bearing a strong resemblance to the evil eye (Figure 1). Due to the progressive cardiopulmonary failure, we therefore performed percutaneous venoarterial extracorporeal membrane oxygenation (VAV-ECMO) using a system that included a centrifugal pump (Rotaflow Centrifugal Pump, Maquet, Germany) and an oxygenator (PLS 2050, Maquet, Germany). The cannula (HLS cannule, Maquet, Germany) sizes were $21 \mathrm{Fr}$ for drainage via the left femoral vein, $17 \mathrm{Fr}$ for infusion via the right femoral artery, and $15 \mathrm{Fr}$ for infusion via the right internal jugular vein in a Y-shaped fashion. He was transferred to our hospital 4 hours later.

On day 3 , surgery was performed through the right atrium and atrial septum and revealed a $75 \times 30 \mathrm{~mm}$ mass attached by a narrow stalk $(10 \mathrm{~mm})$ originating from the atrial septum (Figure 2). ECMO was suspended for 60 minutes during cardiopulmonary bypass and was removed 2 days later. After the application of ECMO, especially after the surgery, the patient's blood pressure gradually stabilized and his lactic acid levels gradually decreased. Troponin I levels rapidly elevated postoperatively and began to decrease from day 5 (Figure 3). Meanwhile, his chest $\mathrm{x}$-rays indicated a significant reduction of the bilateral pulmonary edema after surgery (Figure 4). On day 10 , the patient was discharged from the ICU in good condition and was doing well on outpatient follow-up.

\section{DISCUSSION}

The cardiovascular manifestations of patients with left atrial myxoma strongly depend on tumor size and localization [Layton 2013]. The patient in the present case showed respiratory failure and acute pulmonary edema as the initial signs, which were probably caused by an acute enlargement of the myxoma due to an internal hemorrhage. Unfortunately, the diagnosis of myxoma was overlooked during the initial bedside ultrasound evaluation. This is probably because emergency physicians have less training and experience with ultrasonographic image acquisition and interpretation compared with sonographers or skilled physicians that are trained in echocardiography. In addition, it is challenging to differentiate myxomas from valve vegetations and left atrial clots under severe tachycardia. Therefore, it is important to consider cardiac myxoma in the differential diagnosis of respiratory failure and acute pulmonary edema in emergency departments, especially for patients without a history of heart disease; early consultation with experienced sonologists is recommended.

Despite early positive internal medicine treatment, the patient's condition rapidly regressed into cardiopulmonary failure. During this time, it was discovered that the giant atrial myxoma was the cause and had to be excised immediately; however, the local hospital was unable to carry out the operation. As a result, VAV-ECMO was performed as an alternative intervention. ECMO is a rescue therapy that can stabilize patients with severe hypotension, with or without respiratory failure. In cardiology, the main indications for ECMO include cardiac arrest, cardiogenic shock, post-cardiotomy shock, refractory ventricular tachycardia, and acute management of invasive procedures complications [Guglin 2019]. The use of ECMO as a bridge between the transfer and perioperative periods of patients with acute heart failure in cardiac surgery has been shown to be a viable strategy in recent years; however, it is rarely been used in atrial myxoma cases needing surgical treatment [Deng 2020]. Therefore, the case suggests that patients with atrial myxoma are prone to sudden death; once diagnosed, surgery under the guidance of ECMO should be done if necessary.

\section{Conclusion}

This case illustrates that refractory cardiogenic shock could be the first clinical manifestation of a large left atrial myxoma. Therefore, timely echocardiographic evaluation and surgical removal of the myxoma is recommended, and ECMO could be used as a bridge between the transfer and perioperative periods.

\section{REFERENCES}

Ali MU, Finkel J. 2018. Atrial myxoma. N Engl J Med 379:e26.

Deng L, Xia Q, Chi C, et al. 2020. Awake veno-arterial extracorporeal membrane oxygenation in patients with perioperative period acute heart failure in cardiac surgery. J Thorac Dis 12:2179-87.

Guglin M, Zucker MJ, Bazan VM, et al. 2019. Venoarterial ECMO for adults: JACC Scientific Expert Panel. J Am Coll Cardiol 73:698-716.

Layton S, Ripley DP, Bellenger NG. 2013. Left atrial myxoma. BMJ 347:f4430.

Wedin JO, Kristófi R, Ölander CH, et al. 2020. Cardiac arrest after a transatlantic flight in a patient with a large left Atrial myxoma. CASE (Phila) 4:28-32. 